\title{
Reviews
}

\author{
edited by Philip Barker
}

Eta De Cicco, Mike Farmer and James Hargrave, Using the Internet in Secondary Schools, London: Kogan Page (2nd edn), 2001. ISBN: 0-74943459-7. Softback, $x+192$ pages, $£ 16.99$.

The Internet and its many aspects, including the use of the increasingly media-rich World Wide Web and online communication tools is becoming increasingly central to teaching and learning across the whole educational spectrum. Nevertheless, despite directives such as the National Grid for Learning, which has led to near universal, if still uneven, provision of Internet-enabled technology in schools, and (not always entirely successful) teacher development initiatives such as those provided by NOF (New Opportunities Funding) funded training programmes, effective exploitation of the Internet (and ICT in general) remains a problematic issue in much of our compulsory education system. Of all the things that computers do effectively, it seems, one of the things they continue to do best is to make teachers feel uncomfortable and inadequate ... As such, there continues to be a need for readily available and accessible resources that offer support for those who lack confidence in the use of ICT. This second edition of the book sets out to do precisely this, updating the original 1998 edition (which was reviewed in $A L T-J, 8(3), 109-10$ ) to encompass recent technological developments and changes to the National Curriculum and offer a 'practical, down-to-earth and straightforward guide' to using the Internet in secondary schools.

The book is divided into two main sections: 'Using the Internet in Schools' and 'Curriculum
Activities'. The first focuses predominantly on the Internet as a source of information and resources, a publishing tool and a means of supporting communication and collaborative working practices. The second focuses on the use of the Internet in the curriculum and provides brief but useful introductions to the use of the Internet in teaching a broad range of curriculum areas along with a number of suggested activities and lessons for each.

The first section is divided into four chapters, each following a common format of discussion, examples and links to useful online resources. Chapter 1 offers basic tips on using the Internet and covers Web browsers (with specific focus on Internet Explorer and Netscape), explanations of the various components of URLs, or Web addresses, 'bookmarking' and 'favourites', downloading files from the Web and a discussion of online safety issues. Common to the rest of the book is the friendly, informal tone used to discuss technical issues and the accessible explanations of Internet jargon and terminology. Useful suggestions for using the Web are provided throughout along with screen shots to illustrate more difficult concepts. A minor criticism, however, is that some of the areas dealt with, such as creating and managing 'bookmarks', may have benefited from a more detailed description of the procedures involved.

Chapter 2 concentrates on using the Web in schools and offers an introduction to school networks, and issues related to connecting to the Internet, including broadband, and the 
development of school websites. The section on networks is necessarily brief but should serve to provide teachers with a basic grasp of the technology and terminology involved. An introduction to HTML and Web authoring are included, along with sensible though passing reference to the use of graphics and other design features. Important issues relating to the motivational and empowering aspects of getting children to publish their own work on the Web are also mentioned. While this book in no way purports to offer a comprehensive guide to website development, it does provide pointers to online resources for those who wish to take this subject further.

Chapter 3, which deals with common internet facilities, concentrates on effective use of search engines and a wide range of Internet-related technologies, including common file formats, plug-ins (or browser add-on programs) and programs for handling a range of media types and file-compression programs and techniques. Of the many skills teachers need to develop in order to exploit the Web, effective searching techniques are perhaps the most important and considerable space is devoted to this subject. Methods for narrowing searches using Boolean operators are given alongside useful strategies for developing useful search strings. It is surprising, however, that no mention is given to the use of inverted commas to allow searches on phrases. The chapter provides a veritable raft of pointers to search-engine resources, including subject directories and meta search-engines as well as those specific to the UK, although I do feel that more attention could have been given to the need to evaluate Web-based resources for validity and reliability.

The focus of Chapter 4 is on communicating and learning via the Internet and particular emphasis is given to tools that support collaborative working practices, such as e-mail, mailing lists and online conferencing software. The section on mailing lists is of particular use to teachers wishing to exchange views and ideas with others within a community of practice, and links are given to important resources such as the NGR Virtual Teacher Centre and JSCmail. Tools such as these continue to be underused by teachers and the authors are to be commended for drawing attention to them. Opportunities for developing collaborative learning between pupils in different schools (and countries!) via e-mail exchange programmes and videoconferencing are also discussed, although more attention could have been given to strategies for managing such projects.

Section Two of the book consists of introductions to and examples of using the Internet to support the teaching of Mathematics, English, Science, Citizenship, Geography, History, Design and Technology, Modern Foreign Languages, Music, RE and Art and Design. These have been revised since the 1998 versions and tied into specific areas of the National Curriculum for Key Stages 3 and 4. Key Stage 3 activities have also been linked into DfES Schemes of Work. Learning outcomes are clearly stated and brief but useful notes are provided to enable teachers to adapt and plan the activities for use with their own classes. A useful addition to this section would perhaps have been comments and advice from teachers who had used the lesson suggestions outlined in the original edition, but the current selection does seem sound and usable.

Overall, this book is a useful introductory resource for teachers wishing to improve their understanding and use of the Internet for teaching. The language throughout is accessible and terminology is explained in clear and readily understandable terms. Although the treatment that is given to some aspects of the Internet is necessarily brief, explanations and discussions are always supported with links and pointers to appropriate online resources for those who wish to take things further. The first section of the book will be of most use to those teachers with little or no knowledge of the area, although it is likely that many old hands will also learn some new tricks! The second, curriculum-based, section will surely be of use to all teachers interested in using the Internet in their teaching and provides an excellent foundation on which to build. As a final note, the nature of technological innovation is such that any book on the subject is almost certain to be slightly out of date by the time it is published. As such, it would have been useful to have allied this publication with an online resource of its own that could have been maintained and updated more readily. Nevertheless, this is a useful paperbased introduction to the area of using the Internet to support teaching in secondary schools.

\section{John Knight}

Oxford Brookes University 
S. Ketteridge, S. Marshall and $\mathrm{H}$. Fry, The Effective Academic - A Handbook for Enhanced Academic Practice, London: Kogan Page, 2002. ISBN 0-7494-3570-4. Softback, xi+292, pages, $£ 19.99$.

It has been a turbulent twenty-year period. In the early 1980s I was promoted to a senior lectureship in education at an ancient Scottish university. I ran an annual course in University Teaching Methods for new staff and contributed to some postgraduate teaching on an M.Ed. I had learned my trade as an observant apprentice surrounded by silent journeymen. Then things began to change. The death of the quinquennial review, the reduction in grant funding from the UGC and the transmogrification of the academic life and practice - as we knew it at that time - had begun to take form. I had a sense of this cataclysm at the time, in the rush to set up appraisal of academic staff and training for appraisers.

In this excellent book, the editors and contributors tell the reader of the new reality of academic practice. The new reality is that we practise our trade in a billion-pound international business. We are charged with creating wealth for the higher education sector and we operate at a 'digital chalk face'. The harsh reality is that academic competence is a professional requirement that at best is an aspirational objective rather than an achieved state. So what have the editors and contributors to this book attempted to do in order to prepare us better for our new roles?

In fifteen chapters they provide a romp through the issues that confront the potentially competent academic. To do this they start with a review of the 'turbulent environment' that we choose to work in. While they do not tell us all that has happened in twenty years, the contributors identify the 'academic Munros'. 'In fact the book describes the key features of what the keen academic needs to be able to understand and do in order to survive the chilly winds of the academic terrain. So much for a setting - what about the content? In envisaging this book, the editors took a risk in using academic narrative to carry their message. Some fifty contributors tell stories about the student experience, learning from quality assurance, harnessing C\&IT (computing and information technology) and for learning and developing a strategic culture for research. The level of discourse is aimed at the experienced academic who is about to apply for ILT (Institute for Learning and Teaching) membership. It assumes an intrinsic interest in, and an awareness of, the higher education system and how to make the most of opportunities for learners and teachers alike. When confronted with a request such as 'Please comment on the range of ways in which you contribute to making the learning environment effective for learners . . $\therefore$ ILT applicants need not only to reflect on their own practice, but on the academic landscape they work in.

The contributors do not take prisoners. They tell the story as it seems to them. Alan Hurst (from the University of Central Lancashire), writing about 'students with disabilities', concludes that 'institutions will ... also have to ensure that these students are not treated less favourably than others, and that "reasonable adjustments" have been made, including "adjustments" to the curriculum ... institutions need to start looking ar course provision ... and ensure that they are accessible and inclusive . . Ranging from institutional review to $\mathrm{CPD}$ (continuing professional development) and the 'learning organization', the contributors to this book offer practical advice in a series of 'Enhancing Practice' key messages. In Chapter 4, Tom Kennie (Sheffield Hallam University), writing on strategic and operational planning, offers this missive: 'Imagine you were invited into another department which had recently won a national prize for its success in teaching and research ... During your visit, what sort of evidence would indicate to you that the departmental planning process was of value ...?' Does the approach work? Yes, it does. Storytelling within an academic narrative framework locates practice in a theoretical context. The chosen contributors have a good sense of audience as they describe and evaluate our turbulent system effectively. They draw out messages from the narrative that speaks clearly to the intended reader.

Each of the chapters has a focused bibliography and there is a helpful index (well done Kogan Page!). My professional criticism of the book is that in Su White and Hugh Davis's otherwise excellent chapter on 'Harnessing IT for Learning' (Chapter 13, pp. 232-46), the authors do not really take on the principal tacit assumption in the great rush to make use of C\&IT: 'Are the new learners capable of making the most of C\&IT-rich learning opportunities?' In Chapter 3, 'The Student Experience', Rob Shorrock also skips this issue. If I could add my 
reflective perspective, I would like to ask ILT applicants to reflect on the way we can help our 'co-stakeholders' - the students - to start the process of personal development reflection. In this turbulent system, we must not forget the contribution the learners need to make in order to create personal and systems intellectual wealth. As 'co-stakeholders' we need a companion book called 'The Effective Learner'. One contributor concludes his narrative by saying: 'Keep hoping for the unexpected ... a and remember that the reason academics look young is because they keep on learning ...'

Of course there might be one criticism that I should offer. In the pedagogic narrative tradition, the individual writers have different perspectives and exhibit different views of their intended audience. This might irritate some, as the chapters do not seem to hang together very well. I am prepared to take these multiple perspectives as a case of 'different friends' that I might phone to ask for advice. If I were in that awful television programme where I could 'Phone a Friend', I would like there to be a friendship network where I can get the best advice - not just one assertive or opinionated adviser.

Let's not be frightened about the present. Let's learn from others. Buy this book and take it with you in your rucksack as you "bag your academic Munros!'

\section{Ray McAleese}

University of Aberdeen

Note

1. For the non-Scottish reader there are 284 hills in Scotland whose elevation is greater than 3,000 feet. They are known as 'Munros' after the author of an authoritative list. 'Munro-bagging' is a respectable pursuit for walkers and climbers of all ages.

William Bezanson, Performance Support Solutions - Achieving Business Goals through Enabling User Performance, Victoria, BC: Trafford Publishing, 2002. ISBN: 1-55369-467-8. Softback, xiii +354 pages, $\$ 30.00$.

We live in a world in which we are surrounded by a vast array of sophisticated and complex objects. How do these objects tell us what they are and what they can do? The problem is that, the majority of them do not explicitly do this. Therefore, through the processes of early learning and lifelong development (through ongoing learning, research and experimentation) we acquire the 'working knowledge' that we need in order to recognize various classes of object and the behavioural characteristics that each class exhibits. Of course, in an 'ideal' world, all 'man-made' objects, systems, services and products would be so intuitive and obvious that nobody would need any training or instruction in order to understand what they do or how to use them. Sadly, in this respect, we live in a world that is far from ideal. Hence, the growing need for 'performance support solutions' - and, of course, this new book.

The material in Bezanson's book is organized into four main parts (labelled 'A', ' $\mathrm{B}$ ', ' $\mathrm{C}$ ' and ' $D$ ') that together contain a total of twelve chapters. There is also a set of six appendices and a reference section. In the first part of the book (entitled 'Want Better Users?') the author addresses some of the background and introductory issues upon which the remainder of his material is based. Having covered the 'basics' in the first three chapters, in the second section of the book the author now turns his attention to the theme of 'making products obvious'. The underlying 'message' contained in the three chapters that make up this part of the book relates to the importance of 'performancecentred design'. The three chapters that make up the third part of the book cover the important topics of 'marketing', 'performance architecture' and 'implementing performance support solutions', respectively. The fourth part of the book is devoted to a variety of 'advanced topics' such as 'configuration management', 'testing', 'quality assurance' and the development of 'user support environments'. The six appendices that are included, taken together, provide a comprehensive and detailed analysis (and discussion) of making a business case for using a performance support solution within an organization. Although the reference section is not comprehensive, it serves to indicate the flavour of performance support and the directions in which this technology is now moving.

In the first part of the book (Part A), the author gives a good explanation of the rationale underlying performance support (PS) and how this important area has emerged and grown since the early pioneering work of Gloria Gery and Barry Raybould in the 1980 s. As a working definition, on page 18 , Bezanson suggests that performance support is an attribute of product design that enhances user and business 
Ait-j Volume 11 Number 2

performance through a working environment that anticipates the user's goals and needs and helps users achieve them conveniently and effectively'. The goal of PS is therefore 'developing products that enhance user performance with these products' (p. 49). An important principle underlying the design of a performance support system (PSS) is that there should be an 'integrated deployment of products and support' (p. 52). The support is therefore embedded (intrinsically) within the product - thereby reducing the need for any external form of help (such as an instructor, manual or short course). As an illustration of such a PSS, just think of Microsoft's Office 2002 products - which represent an example of a multi-functional, integrated electronic performance support system.

The three chapters that make up the second part of the book (Part B) deal with performancecentred systems, making products obvious and developing performance support solutions, respectively. As I mentioned earlier, the underlying rationale for this part of the book is the concept of performance-centred design (PCD). This is an approach to design in which performance-enabling attributes are built into a product (or system) so that users do not need extrinsic or external forms of support such as training and/or paper-based documentation. Within this part of the book the author introduces, describes and discusses Gloria Gery's nineteen characteristic attributes of a performance-centred system and then deliberates on how these should be deployed within the context of making a product easy and obvious to use - in other words, enabling users to 'achieve business goals'. He illustrates his case with recourse to the extensive experience that he has had with his Palm Pilot personal digital assistant (PDA). The author goes on to suggest that appropriate combinations of the nineteen performance-centred attributes can be used to make a product 'obvious'. He also recommends that suitable auditing techniques should be used to assess the level to which any given product meets this requirement (p. 108). Of course, as well as designing and auditing systems for 'obviousness' (and the presence of embedded PCD attributes), it is also necessary to be able to create performance support solutions ('PSSolutions') that are based on sound PCD practice. This issue is addressed in some depth in Chapter 6. Essentially, this chapter is devoted to introducing and explaining the various stages involved in the author's proposed six-step Performance Support Solution Design and Development Process.

In Part $\mathrm{C}$, the third section of the book, the author turns his attention to the various ways in which PSSolutions might be implemented. In total, about sixty different methods are identified, described and discussed. His rationale for describing so many discrete methods is simply to allow organizations (that are not ready for full performance support) to get started with one or two small techniques and then to expand incrementally to an end vision of fully integrated performance support. Indeed, such a scheme is the essence of Bezanson's notion of 'performance support solutions'. As was mentioned previously, the first chapter in this section is devoted to the topic of marketing the concept of performance support solutions within business organizations. Two important techniques that are described in this chapter are the use of 'demonstrations' and 'prototyping'. These are often used for proof-of-concept purposes. However, the author strongly advocates their use for 'selling' PSSolutions to both clients and colleagues. The next chapter in this section discusses a variety of ideas relating to the design of a 'performance architecture'. At sixty-two pages, this is by far the longest chapter in the book. Some of the important issues that are discussed include system architecture, performance-enhancing features, user-interaction models, knowledge management technologies and human performance technology. On page 200 the author states that 'the biggest choice to make for a PS information architecture is whether to have the user support materials localised or networked'. Naturally, the use of the Internet and/or in-house intranets figures prominently as an architectural component within this chapter. Indeed, the author recommends: 'Design a PSSolution architecture with a view to distributing and dynamically updating user support materials. The Internet provides an obvious medium for this purpose, and should be considered seriously as part of the architecture' (p. 194). Within Chapter 9 (the third and final chapter in this part of the book) the author presents a number of different methods for transforming the architectural and design ideas (presented in the previous two chapters) into concrete implementations of PSSolutions.

The three chapters that comprise the last part of the book (Part D) explore a number of different advanced topics. For example, in Chapter 10, the 
author consolidates and reinforces many of the issues (that were only briefly introduced and discussed in earlier parts of the book) with more in-depth treatments. Chapter 11 then discusses the problems of designing and building performance systems to support multiple applications. In order to overcome these problems the author introduces the concept of a 'user support environment' (USE). According to the author (p. 290), a USE is 'an open standard for integrating a performance support system with more than one application'. In order to facilitate this approach, the author proposes an architecture involving a 'PSS engine', a 'message bus' and a 'protocol' for using this bus to service the applications that make up a given host system. Several examples are given of how the USE protocol could function within the confines of a multi-application performance support environment. The final chapter in this section provides a conclusion to the book. This chapter is very brief (just two pages!); its essential message is the need to convert performance support solution development into an engineering discipline (akin to software engineering).
Overall, I think this book makes a very useful contribution to the growing field of performance support. However, having said this, it is my opinion that many of the ideas expressed in the book are just 'common sense' HCI (humancomputer interaction) and human-factors issues. Indeed, for many years, in my HCI courses, I have introduced both PS (in general) and ERPSS (Event Related Potential Software System) (in particular) as mechanisms to enable people to overcome their innate, natural limitations - both physical and intellectual. Because of its richness of ideas, I will undoubtedly recommend this book to both my colleagues and students. However, as an academic, I have one major 'crab' about this book - its over-emphasis on 'business applications'. In my view performance support is a generic, universal tool that can be applied to all systems and organizations - not just those whose primary concern is 'business'.

\section{Philip Barker}

University of Teesside 